\title{
An observational study in Irish full day care pre-schools to determine nutrition practice
}

\author{
C. Johnston Molloy ${ }^{1}$, C. Corish ${ }^{2}$, J. Kearney ${ }^{2}$, N. Hayes ${ }^{3}$ and C. Glennon Slattery ${ }^{1}$ \\ ${ }^{1}$ Community Nutrition and Dietetic Service, Health Service Executive, Dublin Mid-Leinster, Marlinstown Office Park, \\ Mullingar, Co. Westmeath, Republic of Ireland, ${ }^{2}$ School of Biological Sciences, Dublin Institute of Technology, Kevin Street, \\ Dublin 8, Republic of Ireland and ${ }^{3}$ Faculty of Applied Arts, Dublin Institute of Technology, Rathmines Road, Dublin 6, \\ Republic of Ireland
}

Recommendations exist for the food intake of infants and young children in pre-school child care ${ }^{(1,2)}$. However, pre-school aged children's diets are reported to be low in vitamin A, vitamin $\mathrm{C}, \mathrm{Fe}$ and $\mathrm{Zn}$ and contain high quantities of salt and sugar ${ }^{(1)}$. In Ireland, while 'Food and Nutrition Guidelines for Pre-schools' are available ${ }^{(1)}$, these are not mandatory and, therefore, methods to encourage the provision of nutritious food in this setting must be pursued. The 'Healthy Incentive for Pre-schools' (HIP) project is an ongoing child care intervention in the Midlands of Ireland which aims to develop and evaluate an incentive scheme to improve pre-school nutrition practices. The aim of this study was to obtain baseline data on the nutrition practices of full day care pre-schools for children aged 3 months to 5 years that had agreed to engage in the HIP project ( $n 76$ of 96 eligible pre-schools). One researcher directly observed and recorded all aspects of nutrition practice in each pre-school over a full day using a structured data collection tool developed for the HIP project. A description of all foods offered was recorded using household measures. A photographic food atlas developed for the HIP project, using foods recommended for pre-school children and recipes from the Irish Health Service Executive '3-week menu plan - a resource for preschools', was used to aid data collection ${ }^{(3)}$. Ethical approval to carry out the study was obtained from the Irish Health Service Executive Dublin Mid-Leinster and the Dublin Institute of Technology Research Ethics Committees.

Due to changes in pre-schools' eligibility to participate (as directed by the local pre-school inspection team), or in the service offered, baseline data were collected in fifty-eight pre-schools; private ( $n$ 39), community $(n 19)$. Data were analysed using SPSS for Microsoft Windows (version 15). Of the forty-three pre-schools that reported having a written healthy eating policy; this was visible in only three services. Fifty-four pre-schools reported having a written menu cycle; however, the cycle length varied widely: 3 week $+(n 28) ; 1$ week ( $n$ 13); 2 week $(n 7)$; none ( $n$ 6). The table provides information on the baseline nutrition practices observed in pre-schools engaged in the HIP project.

\begin{tabular}{lrlr}
\hline Nutrition practices $(n$ 58) & $n$ & & Nutrition practices $(n$ 58) \\
\hline Food routinely provided by parents & 50 & Recommended fruit serving outside main meal & 34 \\
Food prepared on premises & 58 & Recommended dairy serving outside main meal & 3 \\
Breakfast routinely offered & 41 & Recommended number meals and snacks offered & 1 \\
Recommended serving sizes offered at main meal & & Food Pyramid top shelf foods offered & 44 \\
Protein & 3 & Cereal bars & 22 \\
Carbohydrate & 15 & Chocolate & 8 \\
Dairy & 3 & Biscuits & 31 \\
Vegetables & 11 & Sweets & 4 \\
Fe rich food & 3 & Cake & 8 \\
Recommended 'family style food service' practiced & 0 & Recommended use of age appropriate utensils & 1 \\
Clearing plates before end of meal/snack & 55 & Provision of plates for all snack times & 9 \\
Child participating in setting/clearing tables & 5 & Baby bottle use over 12 months of age & 52 \\
Child/staff food discussion at mealtimes & 34 & Lidless 2 handled cups 12 -24 months & 4 \\
Child remaining at table for dining period & 11 & Recommended drinks with meals & 24 \\
All food provided in self-service manner & 0 & Recommended drinks at snack times & 5 \\
\hline
\end{tabular}

In conclusion, few pre-schools provided the recommended: serving sizes of the main food groups; number of meals and snacks; or drink types. Provision of foods from the top shelf of the Food Pyramid occurred frequently. The type of 'family style food service' practice varied and few pre-schools provided recommended feeding utensils. The study results support the need for the development of an incentive scheme to improve nutrition practices within the pre-school setting.

This material is based upon works supported by safefood, the Food Safety Promotion Board, under Grant No. [safefood Grant No. 01-2008]; in association with the Health Service Executive, Ireland.

1. Department of Health and Children (Ireland) (2004) Food and Nutrition Guidelines for Pre-School Services. Dublin: Health Promotion Unit.

2. American Dietetic Association (2005) J Am Diet Assoc 105, 979-986.

3. Johnston Molloy C, Corish C, Kearney J et al. (2010) J Hum Nutr Diet 23, 426-427. 